\title{
NASA Robotic Mining Competition 2017 Systems Engineering Report Badger Robotic Mining Team ${ }^{1}$
}

\author{
David Zeugner ${ }^{2}$
}

\author{
University of Wisconsin-Madison
}

\begin{abstract}
Held at Kennedy Space Center, NASA hosts their annual Robotic Mining Competition to challenge college students with designing and fabricating a robot suitable for mining in environments outside Earth. This year, the Badger Robotic Mining Team at UW-Madison competed for its second time in the competition. To prepare, team members continued work on their Robotic Mining Competition (RMC) 2016 miner, and after such prototyping developed a new robot for RMC 2017. The same mining strategy from the previous competition was used for RMC 2017. This paper summarizes the development of various elements of the mining robot.
\end{abstract}

\section{About Competition}

The Badger Robotic Mining Team from the University of Wisconsin-Madison competed in the NASA Robotic Mining Competition at Kennedy Space Center for its second year in May of 2017. The competition challenges students to design and build a mining robot which can operate in environments outside of Earth while also considering costs of space transportation and lack of maintenance in inhospitable areas. To reflect this, competition points were deducted for robot size, weight, power consumption, and dust creation. Furthermore, use of the atmosphere, GPS, air or foam filled rubber tires, hydraulics, pneumatics, and ultrasonic sensors were prohibited.

The mining arena simulates the Moon's environment. A one-foot layer of Blackpoint-1 (abbreviated BP-1, BP-1 is volcanic ash from Arizona) covers the top surface layer, below which lies a layer of gravel. BP-1 is abrasive and unfriendly to electronics, like that of lunar soil. Obstacles were placed in the arena, which included one-foot deep holes and one-foot diameter rocks. Mining robots must collect what material they can and deposit it into a bin elevated 60centimeters from ground level.

\section{Mining Strategy}

The team based their robot, shown in figure 1, after a scraper design for three reasons. First, the scraper is mechanically the simplest to model and fabricate. This translated to a design with less potential for mechanical failure and a reduced need for maintenance. Second, team's design possessed a high material loading capacity. This was also a result of design simplicity. Lastly, scrapers were not used by other teams and thus made it unique to the Badger Robotic Mining team.

\footnotetext{
${ }^{1}$ The team thanks the WSGC for their donation and invitation to present our work at the $27^{\text {th }}$ annual Space Conference.

2 Other contributors include Tashi Atruktsang, Matt Barrett, Vladimir Bouriakov, Sam Broadnax, James Cho, Jimmy Dodge, Michael Hibbard, Zuodian Hu, William Jen, Travis Sheperd, Jordan Wolff, and Shawn Zhou.
} 
The team's scraper consisted of a bin that was lowered to ground level by linear actuators and filled by driving forward, much like a shovel. To dump material, the bin was raised all the way up to spill regolith out its back end. A mounted vibrator assisted with dumping.

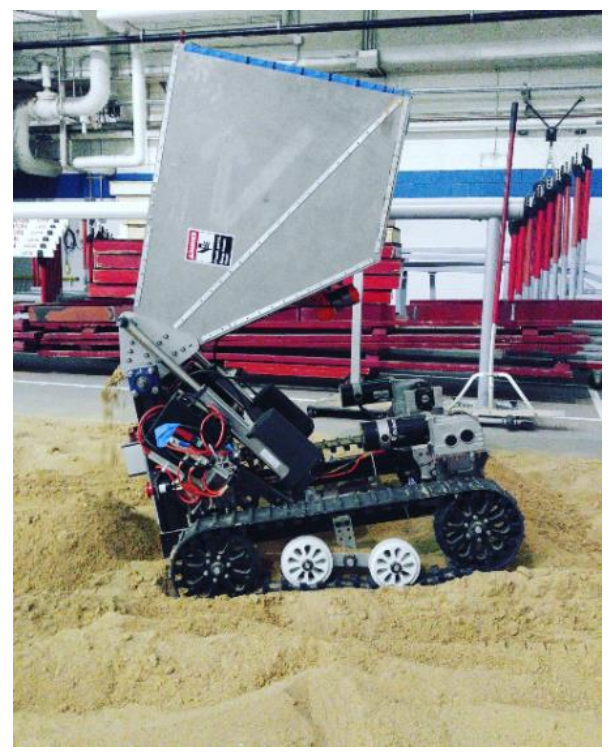

Figure 1. Prototype dumping material.

\section{Development of Robot Components and Systems}

Various parts of the robot were developed: the frame, bin, wheels and treads, motors and mounts, and embedded system. The full rendering of a virtual prototype model can be seen in figure 2 .

Frame. A U-shape frame was chosen for the team's prototype, and persisted all the way through the final design stages. The U-frame allowed for the most flexibility in fitting the mining system onto the robot. The final version of the frame is shown in figure 3.

Our prototype frame material was double 80-20 aluminum. 80-20 provided greater design flexibility since assemblies can be modified quickly. The frame itself weighed 90 pounds. To cut down this figure for our competition 2017 robot, other options were investigated. A steel tube welded chassis was considered first, but the team found better strength to weight ratios with aluminum stock using SolidWorks and ANSYS finite element analysis (abbreviated FEA, used for evaluating structure strength, durability, etc) programs. Stresses studied include ones produced by forces from actuators, material weight inside the bin, and areas of contact between the robot and its environment. 


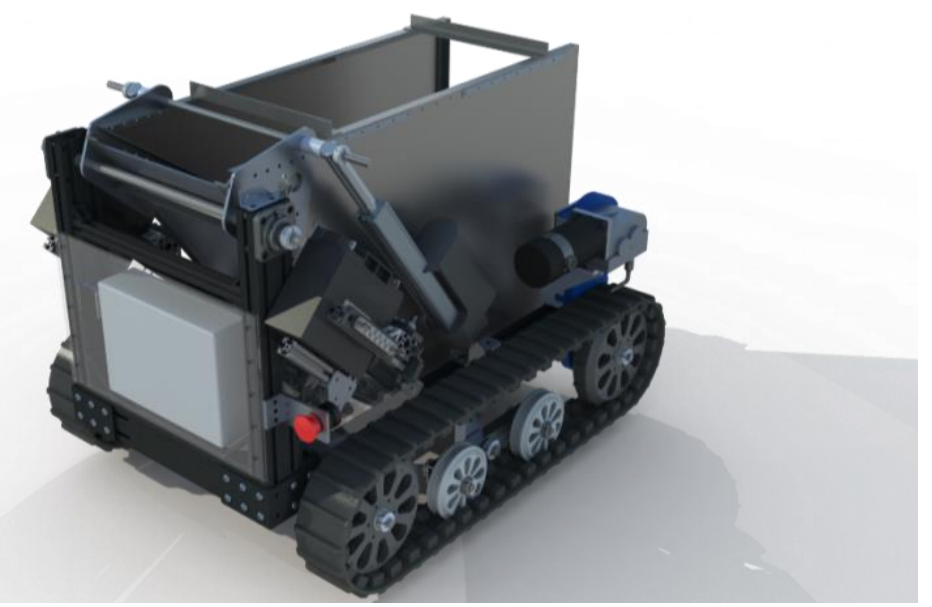

Figure 2. Rendering of prototype with 80-20 frame.

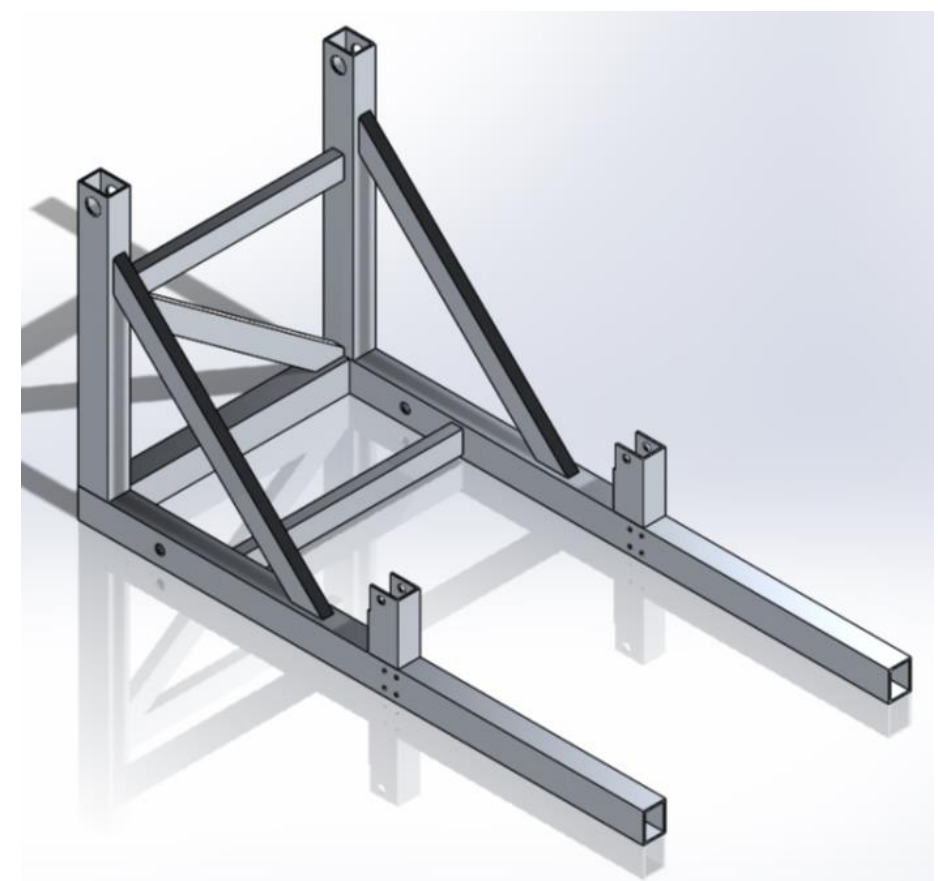

Figure 3. Final iteration of aluminum tube stock frame.

Bin. Design and construction of the bin remained the same throughout prototyping aside from construction materials. The team's original bin consisted of a welded wrought iron frame, a riveted aluminum sheet metal base, and riveted titanium panels. To reduce the weight of this part, aluminum channel replaced the iron channel. The new bin also made use of a composite copper-plastic material in place of titanium, as seen in figure 4. Both materials used for the side panels were salvaged from discarded piles of metal found at our school. The bin skeletal virtual model shown in figure 5 provides a better view of the part as a whole. 

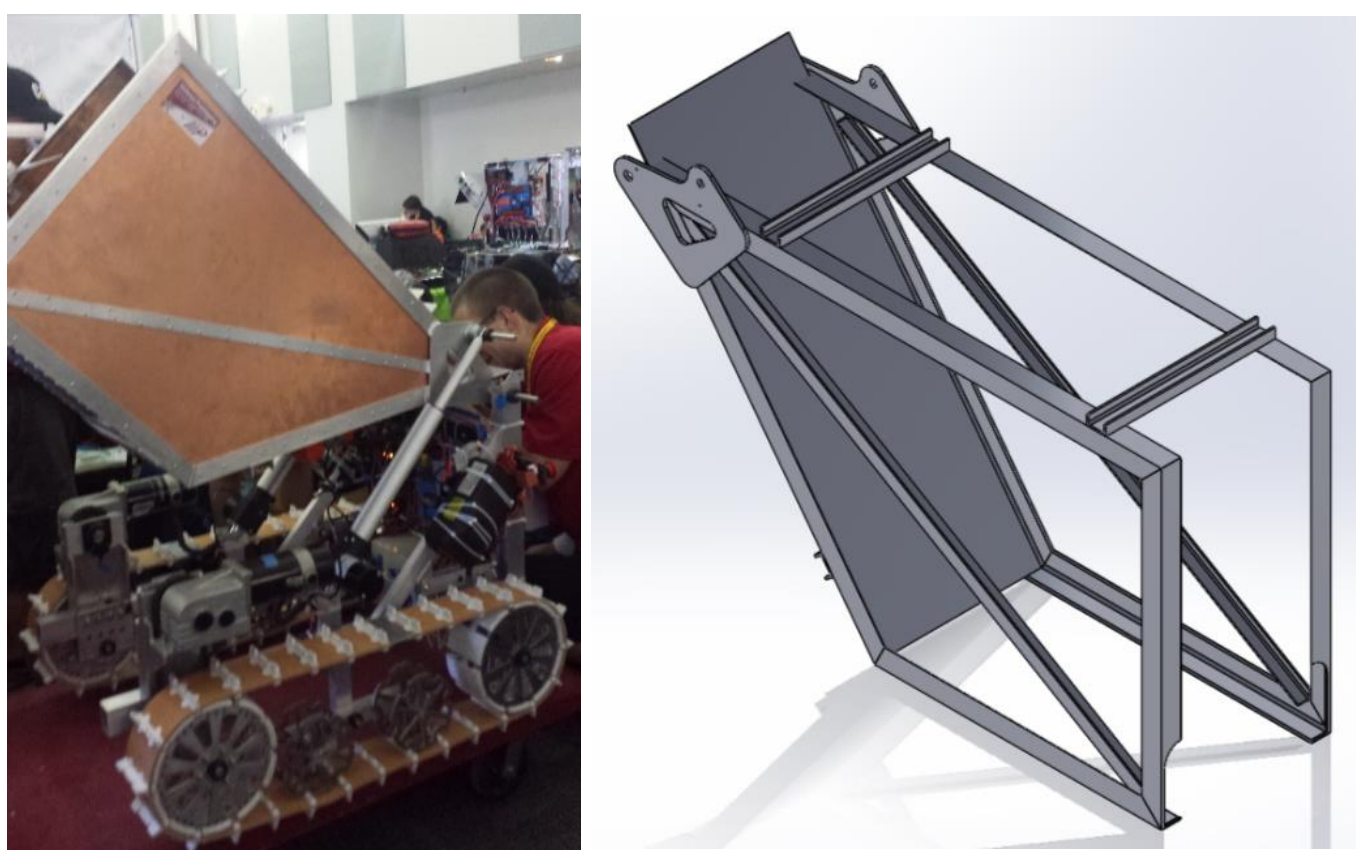

Figures 4 (left) and 5 (right). 2017 Competition bin.

A problem with material collection was discovered through testing. Material was found to collect in front of the bin without entering. To fix the issue, a material displacement mechanism was improvised and added to the bin (figure 6). Initially, it took the form of a broken rocket boat tail mounted to a motor. Its objective was to spin, contact the pile of material, and paddle it to the back of the robot. A more efficient version of the spinner, shown in figure 7, was implemented to the final miner.
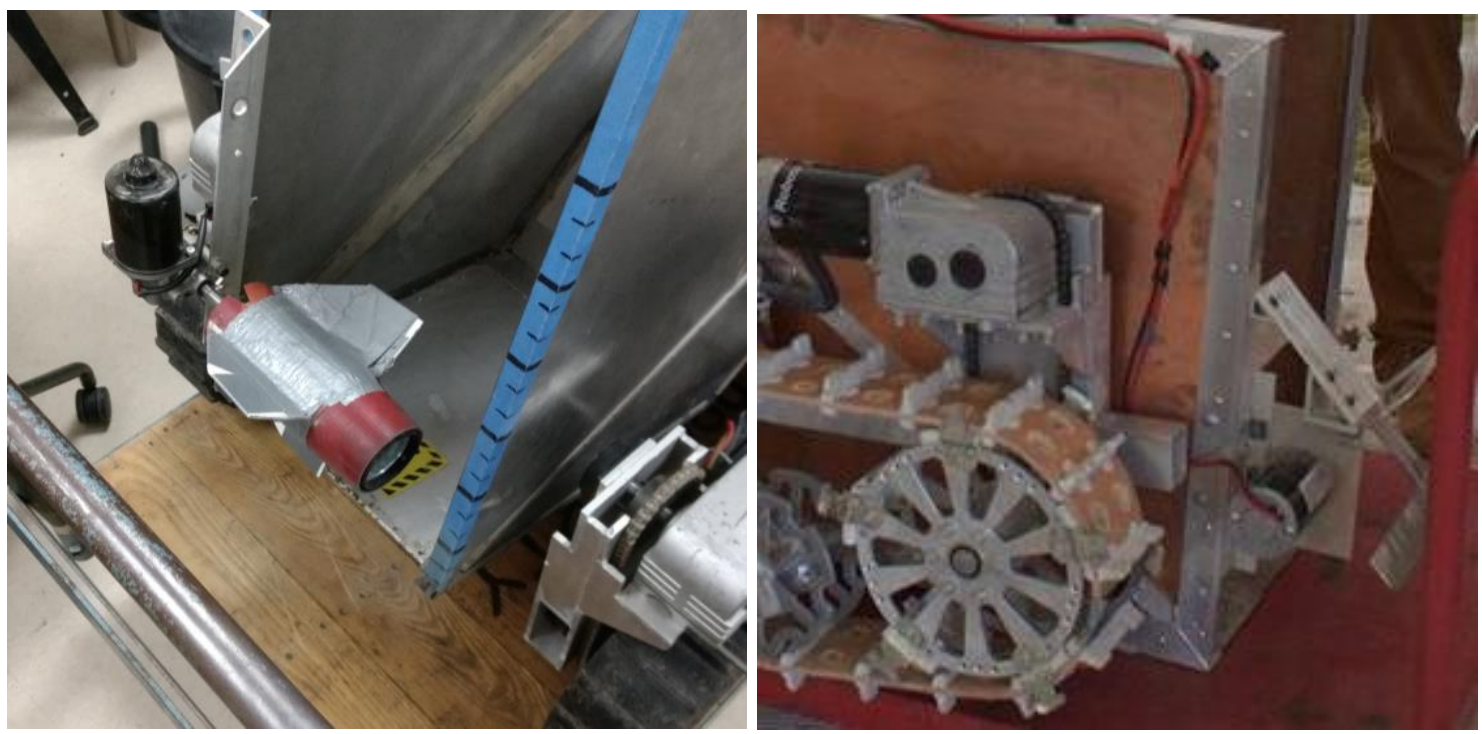

Figures 6 (left) and 7 (right). Improvised and final material displacers.

Wheels and treads. Treads were chosen for their higher traction, necessary for scrapers. The team's prototype treads were acquired by SuperDroid Robots Inc and are shown in figure 8. 


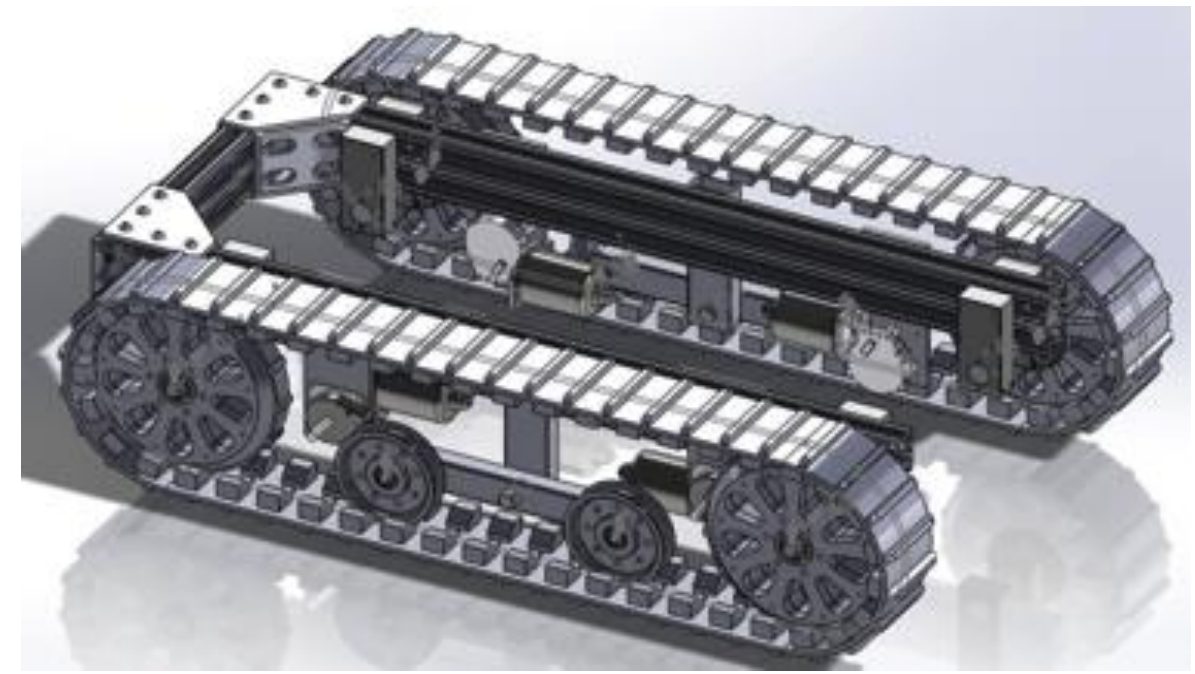

Figure 8. Older prototype powertrain assembly with SuperDroid wheels and treads.

Four free-spinning idler wheels fitting the SuperDroid treads were machined from polyethylene blocks. Their purpose was to more firmly plant the tread onto the ground. Each idler wheel was lowered about a sixteenth-inch below the main wheels to improve the robot's ability to turn without sacrificing significant traction.

New, lighter wheels and treads were developed for the final miner utilizing 3D printed nylon parts (figures 9 and 10). The custom prints were produced by Midwest Prototyping, an additive manufacturing company, and purchased by the team at a discount price.
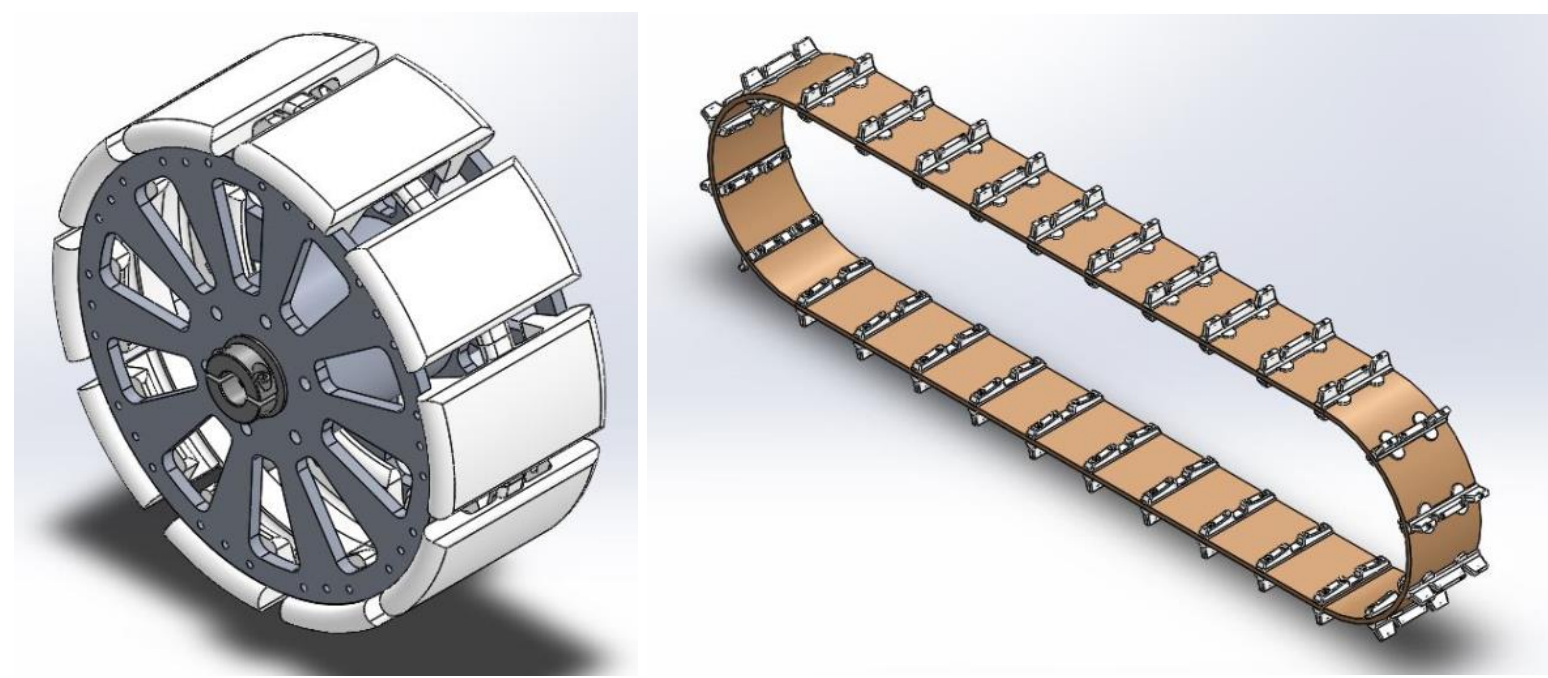

Figures 9 (left) and 10 (right). Final assemblies of wheels and treads.

Motors and mounts. Two discarded wheelchair motors, were acquired from the Wisconsin Electric Machines and Power Electronics Consortium, an on-campus UW-Madison organization. A SolidWorks model of one is shown in figure 11. New mounts were designed and fabricated to fit the motors. A model of one is shown in figure 12. The team determined the best place to house the motors would be towards the front of the robot to improve balance during material dumping. The mounts overhung the motors above the front wheels, the only location with available space. 


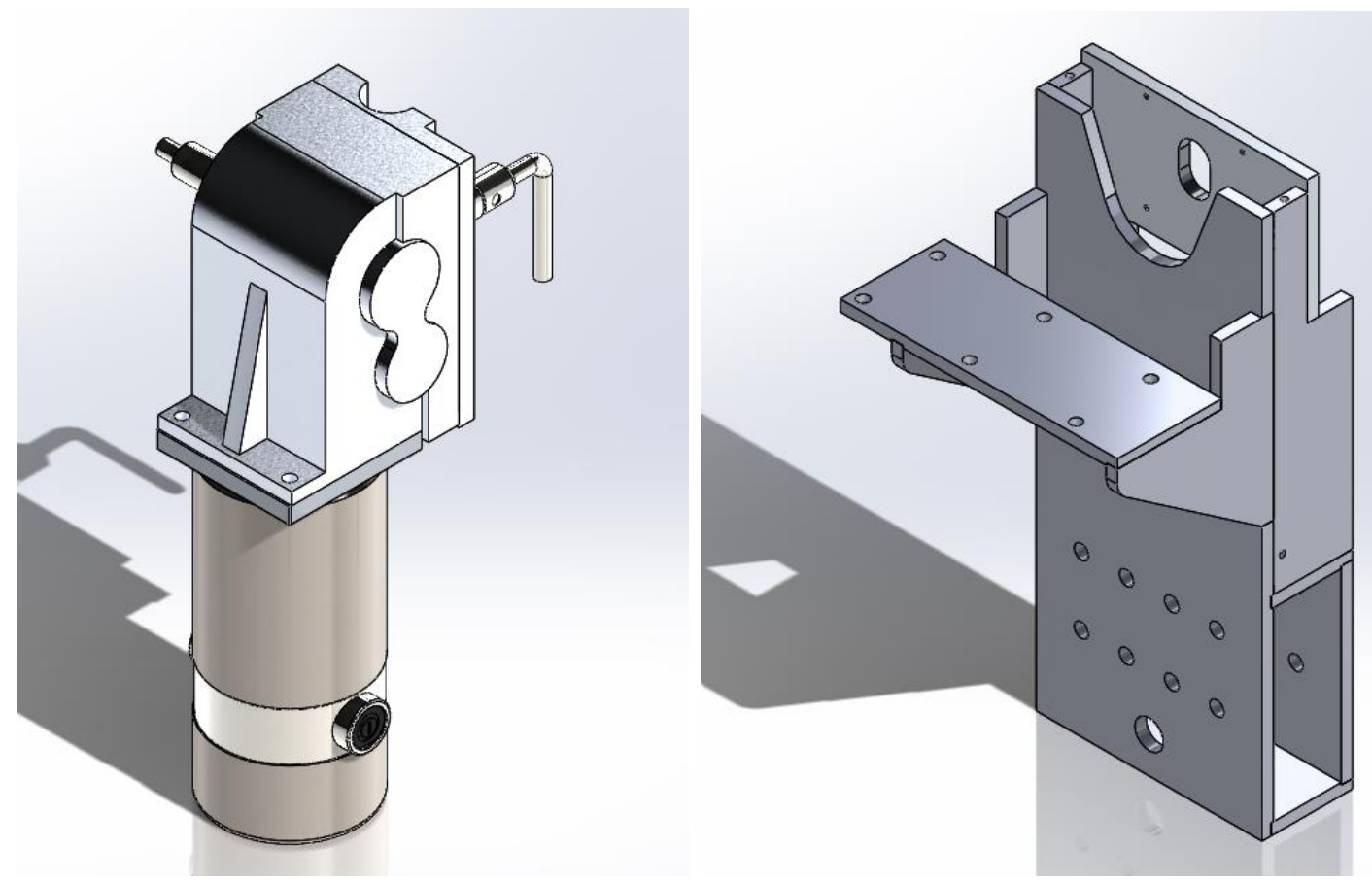

Figures 11 (left) and 12 (right). Motor and mount models.

Embedded system. Both the prototype and final robot used two 24-volt batteries and one 12volt battery. A 24-volt battery powers both wheelchair motors, while the other 24-volt battery powers both actuators and a vibrator mounted on the underside of the bin. The 12-volt battery powers the electronics controlling the robot's motors, actuators, and vibrator. Power consumption was measured by a power logger wired to each battery.

Talon motor controllers were used for each motor and actuator. The commercial motor controllers were necessary to link the motors and actuators to the robot's onboard computer. Control Area Network (CAN bus) packages were selected by the team for coding implemented to the onboard computer.

The embedded processing system consisted of a Raspberry Pi 3 Model B and a Tiva C LaunchPad. The Raspberry Pi acted as the main computer and communicated directly with the LaunchPad. The LaunchPad communicated between the Raspberry Pi and motor controllers.

The team controlled the miner manually using a PlayStation 3 controller synced to a laptop inside a trailer outside of the competition arena. The laptop was linked to a wireless router through an ethernet connection, which was external to the robot but inside the arena. The robot received commands from the wireless router through a Wi-Fi connection. A diagram roughly communicating the connections between each major component of the embedded system is shown in figure 13. 


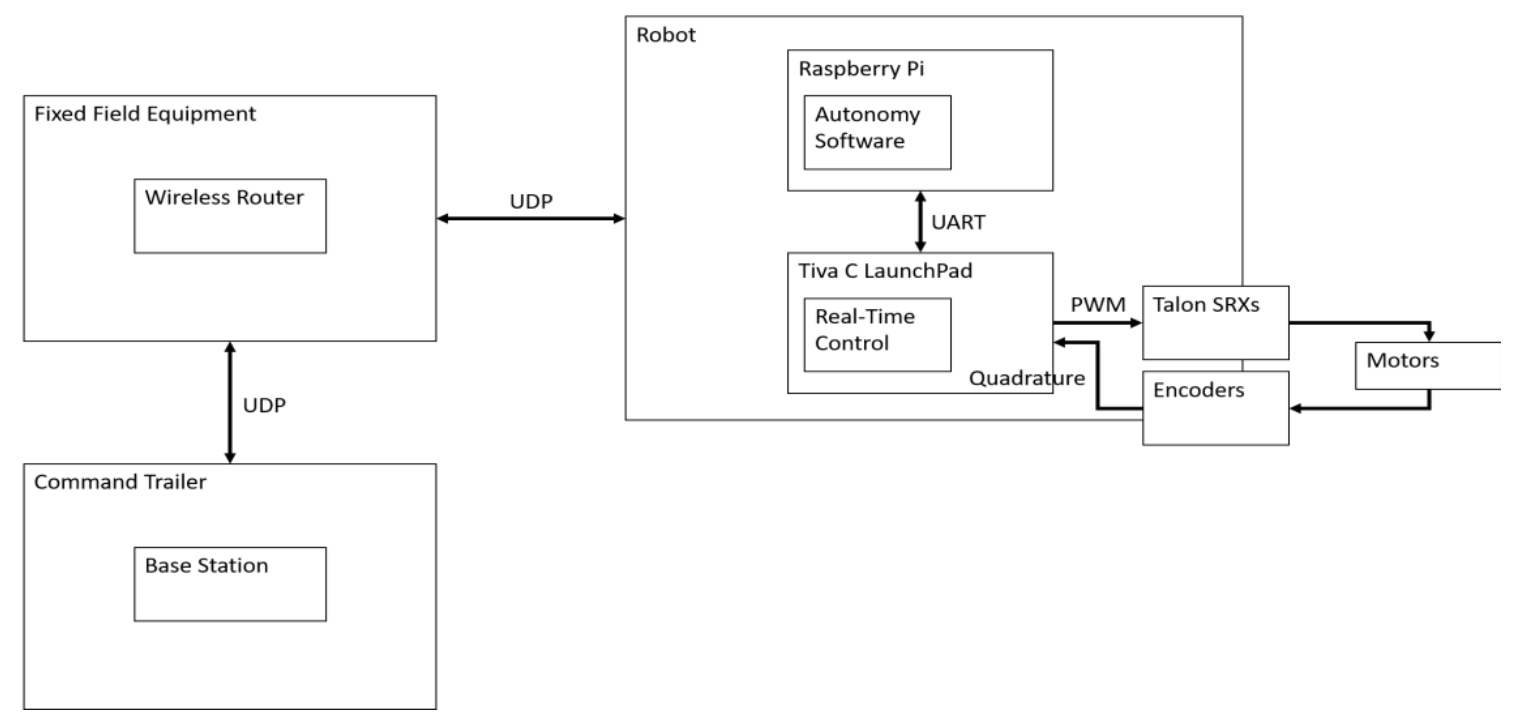

Figure 13. Embedded State Diagram

\section{RMC 2017 performance.}

All systems worked successfully at competition aside from one failure. The team discovered an issue of material clumping between the new wheels and treads. This peeled the treads away from the wheels, resulting in the treads slipping off early in competition runs and an immobilized robot. No material was collected each competition run, however on the first run the bucket was lowered to its digging level, and for the short amount of run time the robot was working properly, about 15 kilograms of material was picked up. Without the treads issue, the team was confident in our design's ability to collect and dump a high quantity of material.

Future modifications to the design include treads that could better shed material that might clump between the wheel and tread. This would most likely involve substitution the solid tread material with a course wire mesh. With more testing, other points of improvement would be identified. The robot's overall weight measured between one and two kilograms shy of the weight limit. Further simulations should be done to further reduce material amount of various robot sections where less material strength is acceptable.

Regardless, students gained experience working with commercial modelling and simulation programs like SolidWorks and ANSYS, machining, and improved other valuable engineering skills. Participation proved to be a useful addition to members' engineering education. 